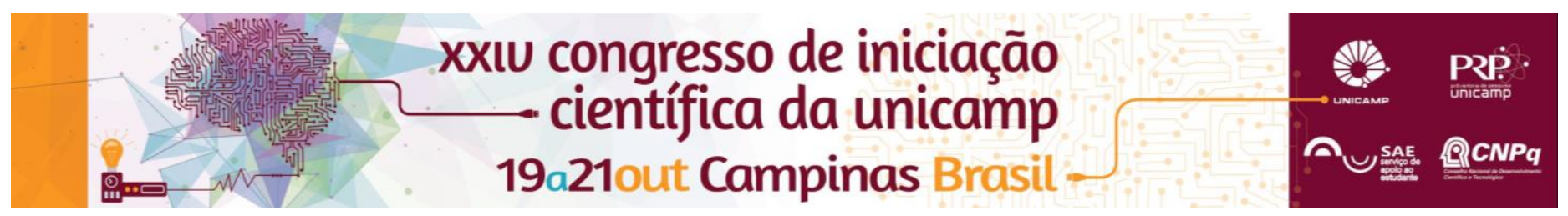

\title{
Robust Model Predictive Control for Trajectory Tracking by an Unmanned Ground Vehicle - An LMI Approach.
}

\author{
João L. B. Martins*, Janito V. Ferreira.
}

\begin{abstract}
Autonomous vehicles are becoming closier to people's reality. Navigation is a great concern for this robotic system. In this paper, we present a control technique for trajectory tracking by the autonomous vehicle. We designed an augmented vehicle model composed by the bycicle model dynamics and a simplified steering model. For maintaining the vehicle in the desired trajectory, a Robust Model Predictive Control (RMPC) technique was implemented using Linear Matrix Inequalites (LMIs) to control the lateral dynamics of the vehicle. Simulation tests were realized using the Double Lane Change maneuver.
\end{abstract}

\section{Key words:}

Unmanned Ground Vehicle, Robust Model Predictive Control, Trajectory Tracking.

\section{Introduction}

The autonomous vehicles, or unmanned ground vehicles, are already a reality in the streets. An automatic driven system is considered the solution for many problems in the cities, as traffic and pollution. In this scenario, the present paper aims to develop a control technique to maintain the vehicle tracking a desired trajectory with minimum distance error. To accomplish this, and regarding the existence of uncertainties between the model and the real vehicle, a Robust Model Predictive Control was implemented and simulated in the Matlab. The assumptions, development and results of this technique can be seen in the next section.

\section{Results and Discussion}

The concept of the Model Predictive Control is to, using the system's model, make predictions of its future outputs and compare with its desirable states to, via an optimization problem, calculate the optimal input to be applied in the current time. Therefore, a good model of the system is required. With that in mind, we propose an augmented vehicle system, combining the bicycle dynamics with a simplified steering model. For trajectory tracking, usually, the vehicle dynamics is represented by the errors between the vehicle center of gravity (CG) and the trajectory, as shown in the figure below:

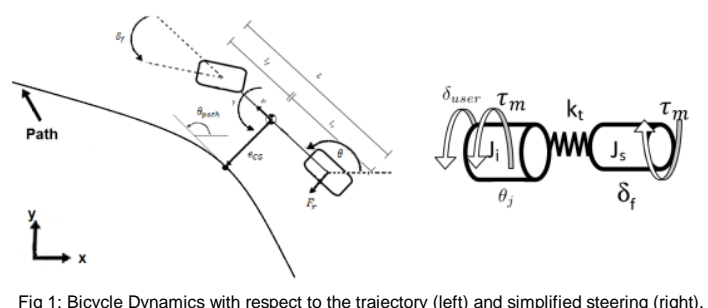
Using small angles approximation, considering a constant longitudinal velocity and a flat plane, the state space for the system model is derived and shown below:

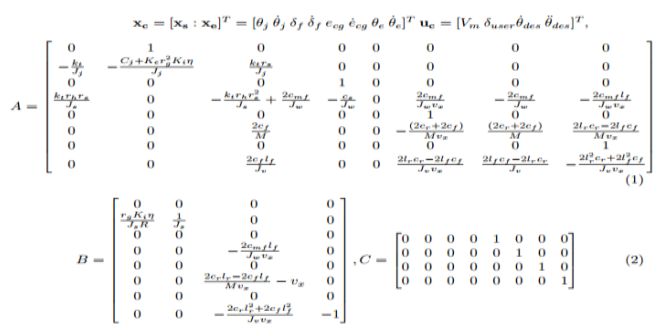

Taking into account mass uncertainties in a real model, a Robust Model Predictive Control technique is presented. For the cost function, we used other augmented system, consisted of the vehicle system and the trajectory dynamics.

$$
\begin{gathered}
J_{\infty}[k]=\sum_{i=0}^{\infty}\left\{\left\|C x[k+i \mid k]-C_{r} x_{r}[k+i]\right\|_{Q_{1}}^{2}+\|u[k]\|_{R}^{2}\right\} \\
J_{\infty}[k]=\sum_{i=0}^{\infty}\left\{\|\tilde{z}\|_{Q_{1}}^{2}+\|u[k]\|_{R}^{2}\right\}
\end{gathered}
$$

We considered a polytope with two vertices to represent our uncertain system. Also, we considered an upper bound for the cost function, which derived the LMIs conditions, for an infinite prediction horizon.

$$
\left[\begin{array}{cc}
Q & \tilde{x}[k \mid k] \\
\tilde{x}[k \mid k]^{T} & 1
\end{array}\right] \geq 0\left[\begin{array}{cccc}
Q & 0 & 0 & (\tilde{A} Q+\tilde{B} Y) \\
\star & \gamma I_{R} & 0 & R^{1 / 2} Y \\
\star & \star & \gamma I_{Q_{1}} & Q_{1}^{1 / 2} \tilde{C} Q \\
\star & \star & \star & Q
\end{array}\right] \geq 0
$$

The result obtained by the controller when executing a double lane change maneuver can be seen below:

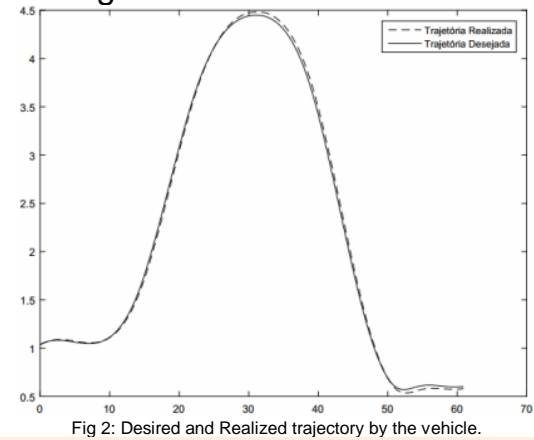

Conclusions

The paper presented a technique based on LMIs for Robust Model Predictive Control of an autonomous vehicle. The technique presented a good behavior in simulations for different values of mass. To increase the complexity and to become more reliable, future studies should add input saturation.

${ }^{1}$ Garcia, O., J. V. Ferreira, and A. Miranda Neto. "Design and simulation for path tracking control of a commercial vehicle using MPC." Robotics: SBRLARS Robotics Symposium and Robocontrol (SBR LARS Robocontrol), 2014 Joint Conference on. IEEE, 2014.

${ }^{2}$ Kothare, Mayuresh V., Venkataramanan Balakrishnan, and Manfred Morari. "Robust constrained model predictive control using linear matrix inequalities."Automatica 32.10 (1996): 1361-1379.

${ }^{3}$ Maciejowski, Jan Marian. Predictive control: with constraints. Pearson education, 2002. 